\title{
Computation of Optimal Current References for Flux-weakening of Multi-Phase Synchronous Machines
}

\author{
Li Lu ${ }^{(1)}$, Bassel Aslan ${ }^{(1)}$, Luc Kobylanski ${ }^{(2)}$, Paul Sandulescu ${ }^{(1)}$, F.Meinguet ${ }^{(1)}$, Xavier Kestelyn ${ }^{(1)}$ and Eric Semail $^{(1)}$ \\ (1) Arts et Metiers Paristech - L2EP, 8 Bd Louis XIV, 59046 Lille France \\ (2) VALEO-EEM, 2 rue André Boulle, 94000 Créteil France \\ Corresponding author : Xavier.Kestelyn@ensam.eu
}

\begin{abstract}
Multi-phase synchronous machines are more and more used in specific applications where high power density, low bus voltage, wide speed range and fault-tolerant capabilities are required. Due to the high number of degrees of freedom, multiphase machines are difficult to optimally operate in fluxweakening zones. This paper proposes a technique to numerically compute optimal current references that can be used for feed-forward flux-weakening techniques in order to exploit the maximum machine performances for given DC bus voltage and current limits. The proposed technique is applied to a five-phase permanent magnet synchronous machine specifically developed for an automotive application.
\end{abstract}

\section{INTRODUCTION}

Multi-phase synchronous machines are more and more used in specific applications where high power density, low DC bus voltage, wide speed range and fault-tolerant capabilities are required [1]. The automotive context offers a perfect example where multi-phase permanent magnet machines are now used. Low voltage DC bus, around $60 \mathrm{~V}$, insures an easy electrical security and reduces constraints on the Battery Management Systems (BMS). In the range from 10 to $20 \mathrm{~kW}$, three-phase machines are not well suited because the resulting phase currents would be too large. Consequently, multi-phase machines become a good solution to reduce phase currents since it is possible to split the power into more than three phases. In MHYGALE project, proposed by Valeo and funded by ADEME, a five-phase permanent magnet prototype machine has been designed in order to obtain a mild hybridization solution affordable for the greatest number with a significant impact on worldwide $\mathrm{CO} 2$ emissions [2]. This solution, based on a high-power belt-driven electrical machine, offers the start-stop concept, a regenerative braking and torque assistance. The torque boost makes possible to downsize the Internal Combustion Engine (ICE) along maintaining equivalent performances as provided by a more powerful ICE. The major interest from the point of view of the car supplier is the possibility to keep the same car architecture since the proposed electrical machine simply replaces the common belt-driven Lundell. A smooth and low cost transition is thus obtained towards mild hybrid vehicles.

Due to the high number of degrees of freedom proposed by multi-phase machines, it is difficult to operate optimally the machine in flux-weakening zones. This paper proposes a technique to numerically compute optimal current references that can be used for feed-forward flux-weakening techniques in order to exploit the maximum machine performances for given voltage and current limits.

Very few papers have addressed the difficult problem of flux-weakening operation of multi-phase motors. Actually, finding a set of current references that maximize the torque density taking into account the voltage limits leads to a difficult problem to write and often impossible to solve analytically, except if using very restrictive assumptions [3][6]. In the context of severe operating conditions, the assumptions that are used in the current literature lead to a partial exploitation of full performances and to a costly oversizing of power components.

Compared with star-connected three-phase machines which are controlled in only one $d q$ subspace, multi-phase machines have to be simultaneously controlled in several orthogonal $d q$ subspaces. Actually, multiphase drives offer the opportunity to increase the torque density by adding spatial harmonics in the magnetic field. Working with Park variables, it means that, for given DC bus, several sets of (id, iq) currents have to be determined in order to get the maximum torque that can be produced by each current harmonic (saliency torque included).

It is easy to analytically compute current references in the case of non-salient multi-phase permanent magnet machines working below the voltage and current limits [7]. However, it becomes very difficult to find an analytical expression of required currents when the voltage limit has been reached. This difficulty grows when the saliency torque is taken into account.

When the DC bus voltage is completely exploited, id currents have to be increased in order to start the fluxweakening operation. Some authors propose a few heuristic methods to generate the current references but without any guarantee concerning the optimality of the proposed solution. The major problem comes from the fact that when a voltage limit is reached in one $d q$ subspace, it becomes very difficult to know what are the limit reference voltages in the other $d q$ subspaces. In the literature, a simplification is chosen in order to consider as variables of the problem only the magnitudes of the different $d q$ vectors, disregarding their phase angles [5],[8]. This choice corresponds to the worst case that can be encountered. Consequently, it leads to use the 
DC bus under its maximum, which is not acceptable in low voltage applications.

This paper proposes then a method in three steps to compute each set of $(i d, i q)$ current references according to the expected torque and mechanical speed under constraints of voltage and current limits. The method uses classical optimization tools offer by commercial software. Compared to solutions found in the literature, less assumptions are taken and the DC bus is better exploited. A feed-forward based flux-weakening scheme of a five-phase permanent magnet machine is presented in order to validate the optimality of the proposed technique.

\section{MACHINE MODELLING}

Equations (1) to (5) model the considered star-connected five-phase permanent magnet synchronous machine in two $d q$ subspaces (associated with the first and the third air gap harmonics), under the following assumptions:

- $\quad$ Flux saturation, hysteresis and eddy current losses in the iron cores are not taken into account

- The slot effect is not considered

- Only harmonics one and three are considered.

In (1) to (5), variables are:

- $\quad v_{1 d}, v_{1 q}, v_{3 d}, v_{3 q}$ and $i_{1 d}, i_{1 q}, i_{3 d}, i_{3 q}$ the $d$ and $q$ axis voltages and currents associated with the first and the third air gap harmonics

- $\quad R$ the phase resistance

- $\quad L_{1 d}, L_{1 q}, L_{3 d}, L_{3 q}$ the $d$ and $q$ inductances associated with the first and the third air gap harmonics

- $\quad p$ the number of pole pairs

- $\quad \Omega$ the mechanical speed of the shaft

- $\quad \psi_{1 f}, \psi_{3 f}$ the flux created by the permanent magnets along $d$ axis associated with the first and the third air gap harmonics

- $\quad T_{e m l}$ the electromagnetic torque produced by the first air gap harmonic

- $\quad T_{\text {em3 }}$ the electromagnetic torque produced by the third air gap harmonic

- $\quad T_{e m}$ the total electromagnetic torque

$$
\begin{aligned}
& v_{1 d}=R i_{1 d}+L_{1 d} \frac{d i_{1 d}}{d t}-p \Omega L_{1 q} i_{1 q} \\
& v_{1 q}=R i_{1 q}+L_{1 q} \frac{d i_{1 d}}{d t}+p \Omega\left(L_{1 d} i_{1 d}+\sqrt{\frac{5}{2}} \psi_{1 f}\right) \\
& v_{3 d}=R i_{3 d}+L_{3 d} \frac{d i_{3 d}}{d t}+3 p \Omega L_{3 q} i_{3 q} \\
& v_{3 q}=R i_{3 q}+L_{3 q} \frac{d i_{3 q}}{d t}+3 p \Omega\left(L_{3 d} i_{3 d}+\sqrt{\frac{5}{2}} \psi_{3 f}\right) \\
& T_{e m 1}=p\left[\left(L_{1 d}-L_{1 q}\right) i_{1 d} i_{1 q}+\sqrt{\frac{5}{2}} \psi_{1 f} i_{1 q}\right] \\
& T_{e m 3}=p\left[3\left(L_{3 d}-L_{3 q}\right) i_{3 d} i_{3 q}+3 \sqrt{\frac{5}{2}} \psi_{3 f} i_{3 q}\right] \\
& T_{e m}=T_{e m 1}+T_{e m 3}
\end{aligned}
$$

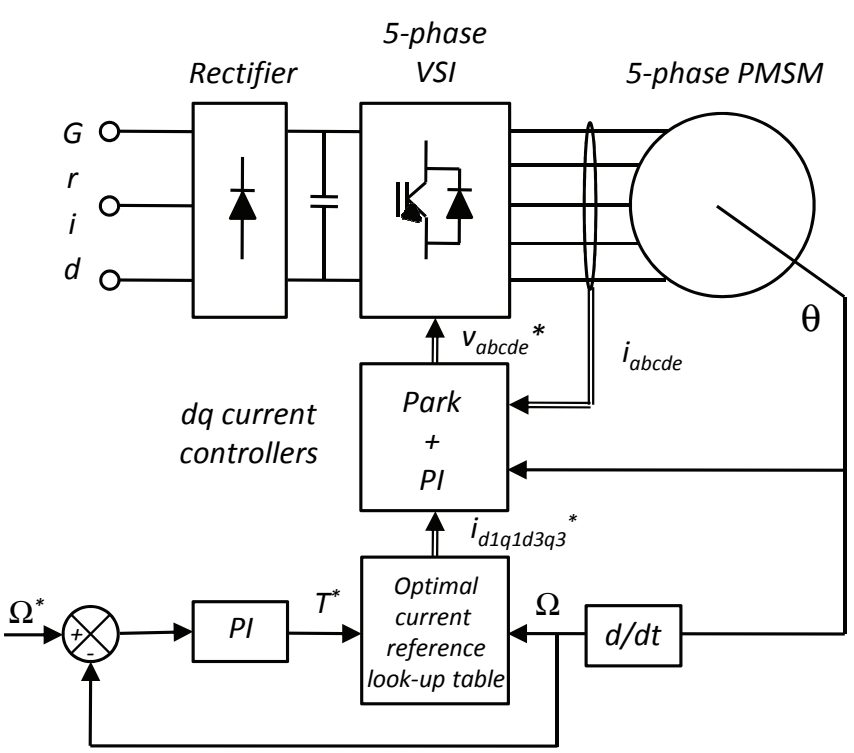

Fig. 1. Proposed control scheme

\section{CONTROL STRATEGY}

\section{A. Implemented Control scheme}

Fig. 1 shows the implemented control scheme. The speed is controlled using a PI controller and generates a torque reference. From this torque reference and the actual speed, $d q$ current references are generated. The speed is estimated from the position measured by a synchro resolver and $d q$ current reference data are stored in 2D look-up tables. Current references are compared to $d q$ currents, estimated from the measured real currents transformed by $T$ expressed by (8). All current data are obtained using numerical computations discussed in the next section.

Finally, $d q$ currents are controlled by PI controllers and, using inverse transformation of $T$, generate voltage references which are sent to a five-phase voltage source inverter.

$$
T=\sqrt{\frac{2}{5}}\left(\begin{array}{ccccc}
1 / \sqrt{2} & 1 / \sqrt{2} & 1 / \sqrt{2} & 1 / \sqrt{2} & 1 / \sqrt{2} \\
\sin (p \theta) & \sin (p \theta-2 \pi / 5) & \sin (p \theta-4 \pi / 5) & \sin (p \theta+4 \pi / 5) & \sin (p \theta+2 \pi / 5) \\
\cos (p \theta) & \cos (p \theta-2 \pi / 5) & \cos (p \theta-4 \pi / 5) & \cos (p \theta+4 \pi / 5) & \cos (p \theta+2 \pi / 5) \\
\sin 3(p \theta) & \sin 3(p \theta-2 \pi / 5) & \sin 3(p \theta-4 \pi / 5) & \sin 3(p \theta+4 \pi / 5) & \sin 3(p \theta+2 \pi / 5) \\
\cos 3(p \theta) & \cos 3(p \theta-2 \pi / 5) & \cos 3(p \theta-4 \pi / 5) & \cos 3(p \theta+4 \pi / 5) & \cos 3(p \theta+2 \pi / 5)
\end{array}\right)
$$

\section{B. $\quad$ Comparison to existing methods}

The computation of the current references in the fluxweakening zone is a difficult task. In order to be able to find analytical solutions to this problem, classical assumptions that are found in the literature are summarized:

- Stator resistances are neglected

- $\quad$ Saliency torque is not exploited $\left(i_{d}\right.$ currents are set to zero below the base speed)

- To determine the maximum required voltage, it is assumed that harmonic voltage references (one and three in our case) reach their peak values in the same time instant, i.e. only magnitudes are considered disregarding the phase angles 
All these assumptions lead to a non-optimal exploitation of intrinsic performances. Since it is very difficult to obtain an analytical solution to the problem without considering the former assumptions, in the next section a numerical computation of optimal references is proposed.

\section{Computation of optimal dq current references}

The optimal current reference data, stored in look-up tables, are obtained in three steps. All steps use numerical optimization tools (fmincon from the MATLAB Optimization Toolbox in this paper) which make possible to maximize or to minimize a function under constraints. No other assumptions than the ones proposed in section II are considered.

The first step consists in finding the speed limits and to compare them to the desired maximum speeds (negative and positive). When the computed speed limits are superior to the maximum desired speeds, the second step is started. The function to be maximized is expressed by (9).

$$
\max \Omega \text { such that }\left\{\begin{array}{l}
\left(v_{a} \text { to } v_{e}\right) \leq \frac{V_{\text {bus }}}{2} \\
\left(i_{a} \text { to } i_{e}\right) \leq I_{\max }
\end{array}\right.
$$

The second step consists in computing the boundaries of torque-speed characteristics (negative and positive). The function to be maximized is expressed by (10).

$$
\max T_{e m} \text { such that }\left\{\begin{array}{l}
\left(v_{a} \text { to } v_{e}\right) \leq \frac{V_{b u s}}{2} \\
\left(i_{a} \text { to } i_{e}\right) \leq I_{\max }
\end{array}\right.
$$

The third and last step consists in finding several $\left(i_{d}, i_{q}\right)$ sets for torque and speed which are comprised between torque and speed limits. The function to be maximized is expressed by (11).

$$
\min C_{\text {losses }} \text { such that }\left\{\begin{array}{c}
\left(v_{a} \text { to } v_{e}\right) \leq \frac{V_{\text {bus }}}{2} \\
\left(i_{q} \text { to } i_{e}\right) \leq I_{\max } \\
C_{\text {losses }}=R\left(i_{d 1}{ }^{2}+i_{q 1}{ }^{2}+i_{d 3}{ }^{2}+i_{q 3}{ }^{2}\right) \\
T_{e m}=\left(T_{e m}\right)_{\text {ref }}
\end{array}\right.
$$

\section{SIMULATION AND EXPERIMENTAL RESUlTS}

\section{A. Machine parameters}

The five-phase machine is a permanent magnet synchronous machine prototype which has been developed for the project MHYGALE proposed by Valeo. All parameters are summarized in Table I. It has to be noticed that due to its particular structure, the machine possesses a small saliency only related to the third harmonic.

Fig. 2 shows the boundaries of torque-speed characteristics that have been computed using step 2 of the proposed method. The base speed is evaluated at $1500 \mathrm{rpm}$. Below the base speed, the maximum torque is equal to 55.8 N.m.
TABLE I

MACHINE PARAMETERS

\begin{tabular}{|l|c|}
\hline \multicolumn{1}{|c|}{ Parameter } & Value \\
\hline Phase resistance $R$ & $9.1 \mathrm{~m} \Omega$ \\
\hline Inductance $L_{l d}$ & $0.13 \mathrm{mH}$ \\
\hline Inductance $L_{l q}$ & $0.13 \mathrm{mH}$ \\
\hline Inductance $L_{3 d}$ & $0.051 \mathrm{mH}$ \\
\hline Inductance $L_{3 q}$ & $0.041 \mathrm{mH}$ \\
\hline Flux $\psi_{l f}$ & $19.4 \mathrm{mWb}$ \\
\hline Flux $\psi_{3 f}$ & $0.675 \mathrm{mWb}$ \\
\hline Maximum torque $\left(T_{e m}\right)_{\max }$ & $55.8 \mathrm{~N} . \mathrm{m}$ \\
\hline Bus voltage $V_{\text {bus }}$ & $60 \mathrm{~V}$ \\
\hline Maximum phase current $I_{\max }$ & $144 \mathrm{~A}$ \\
\hline Maximum speed $\Omega_{\max }$ & $18000 \mathrm{rpm}$ \\
\hline
\end{tabular}

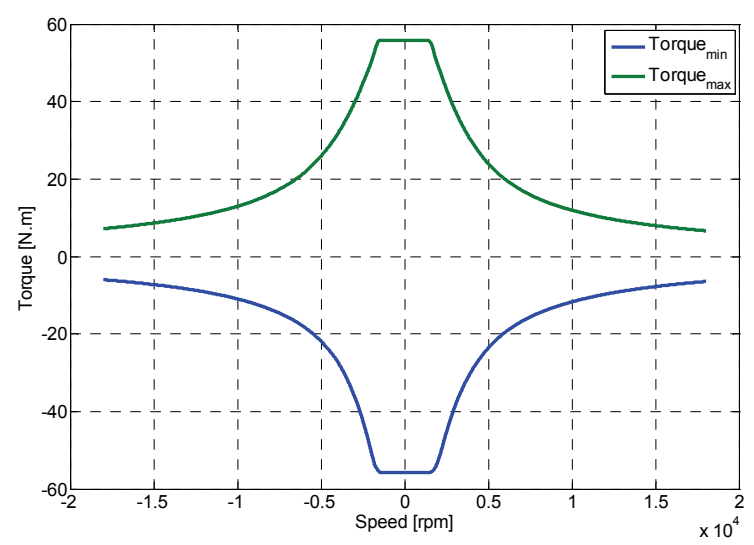

Fig. 2. Computed boundaries of torque-speed characteristics

\section{B. $\quad$ Experimental validations}

The proposed current references have been computed and tested on a five phase PMSM supplied by a five-leg VSI. Fig. 3 shows a snapshot of the experimental setup which has been recently developed for MHYGALE project.

For practical reasons, the DC bus has been reduced to $30 \mathrm{~V}$ and the maximum phase current to $25 \mathrm{~A}$. Under these conditions, the base speed is equal to $98 \mathrm{rad} / \mathrm{s}$, the maximum speed to $127 \mathrm{rad} / \mathrm{s}$ and the maximum torque to 9.6 N.m.

The analysis of the different $i_{d q}$ currents for a given speed and torque is difficult since there are several functioning zones where the objectives are different. The currents' behaviours depend on the mechanical speed (below and above the base speed) and the peak phase currents (before their have reached the maximum and when they have reached the maximum).

\section{Below the base speed $(\Omega<98 \mathrm{rad} / \mathrm{s})$}

$i_{1 d}$ and $i_{3 d}$ are almost equal to zero since there is no need for weakening the machine flux and the machine is quasi non salient. 
- Below the current limit $\left(i_{\text {peak }}<25 A\right)$

To analyse $i_{q}$ currents, please refer to Fig.4, which shows the ratio between $i_{3 q}$ and $i_{1 q}$ for different torque references (from zero to max) below the base speed (for example 50 $\mathrm{rad} / \mathrm{s}$ ). For a torque between 0 and 7.8 N.m, peak phase currents do not reach the maximum value and the ratio $i_{3 q} / i_{1 q}$ is equal to $3 \lambda_{3 f} / \lambda_{1 f}$ (in that case equal to 0.104 ), leading to a given torque under minimum copper losses [7]. Torques $T_{e m l}$ and $T_{e m 3}$, respectively associated with the first and the third harmonics, are both positives.

See Fig. 8 and Fig.9 for experimental results below the base speed and when the peak phase current is below the maximum.

○ At the current limit $\left(i_{\text {peak }}=25 \mathrm{~A}\right)$

When peak phase currents reach the maximum (for a torque equal to $7.8 \mathrm{~N} . \mathrm{m}$ ), ratio $i_{q 3} / i_{q 1}$ progressively falls to 0.156 to fully exploit the current limit (see Fig.5). A negative ratio means that the third current harmonic is minimum when the first current harmonic is maximum, leading to the wellknown case of a third harmonic injection when the first harmonic of a variable has to be maximum. In that case, it has to be noticed that torque $T_{e m 3}$ is negative along with a positive torque $T_{e m l}$. The optimality is then to fully exploit the current limit instead of minimizing the copper losses for a given torque.

See Fig.10 and Fig.11 for experimental results below the base speed and when the peak phase current is equal to the maximum.

\section{Above the base speed $(\Omega>98 \mathrm{rad} / \mathrm{s})$}

$i_{l d}$ and $i_{3 d}$ are different from zero since there is need for weakening the machine flux and the machine is quasi non salient.

\section{- Below the current limit $\left(i_{\text {peak }}<25 \mathrm{~A}\right)$}

As shown in Fig.5 $i_{l d}$ is negative whereas $i_{3 d}$ is positive since it is the optimal way to reduce the peak phase voltage and then keeping the torque constant although the base speed has been reached. The peak phase current grows since $i_{l q}$ stays constant.

See Fig. 12 and Fig. 13 for experimental results above the base speed and when the peak phase current has not been reached.

\section{- At the current limit $\left(i_{\text {peak }}<25 A\right)$}

As shown in Fig.5 and Fig.7, when the peak current reaches his limit, slopes of $i_{d q}$ currents change rapidly in order to shift current harmonics one and three to optimally exploit the current limit. The torque decreases since $i_{q}$ currents cannot be kept as they were before.

\section{CONCLUSION}

This paper presents an method to generate optimal current references in order to get the maximum torque of a multi-phase machine while using at their maximum the bus voltage and the phase currents. In order to validate the proposed method, a salient five-phase permanent magnet machine with non-sinusoidal Electro-Motive Forces has been tested below and in the flux-weakening region. Experimental results prove the effectiveness of the proposed method and show how can be used the extra degrees of freedom offered by multi-phase machines to fully exploit machine's capabilities. Future improvements as high order harmonic injection and feedback scheme to cope with parameter variation are in progress.

\section{ACKNOWLEDGMENT}

This work is part of the project 'MHYGALE' (Mild Hybrid for Generalization) with funding by ADEME (French Agency for Control and Saving Energy) within Valeo Engine Electrical Equipment.

\section{REFERENCES}

[1] Parsa, L.; , "On advantages of multi-phase machines," Industrial Electronics Society, 2005. IECON 2005. 31st Annual Conference of IEEE, vol., no., pp. 6 pp., 6-10 Nov. 2005

[2] Li Lu; Semail, E.; Kobylanski, L.; Kestelyn, X.; , "Flux-weakening strategies for a five-phase PM synchronous machine," Power Electronics and Applications (EPE 2011), Proceedings of the 201114th European Conference on, vol., no., pp.1-7, Aug. 30 2011-Sept. 1 2011

[3] L.Parsa, Kim Namhun, H.A.Toliyat, "Field Weakening Operation of High Torque Density Five-Phase Permanent Magnet Motor Drives", Proceedings of 2005 IEEE International Conference on Electric Machines and Drives (IEMDC2005), pp. 1507-1512, 2005.

[4] D.Casadei, M.Mengoni, G.Serra, A.Tani, L.Zarri, L.Parsa, "Control of a High Torque Density Seven-phase Induction Motor with Fieldweakening Capability', Proceedings of 2010 International Symposium on Industrial Electronics (ISIE2010), pp. 2147-2152, 2010.

[5] E. Levi, D. Dujic, M. Jones, G. Grandi "Analytical Determination of DC-Bus Utilization Limits in Multiphase VSI Supplied AC Drives", IEEE Trans. on Energy Conversion, Vol. 23, No. 2, June 2008, pp.433443.

[6] Song Xuelei; Wen Xuhui; Cong Wei; , "Research on field-weakening control of multiphase permanent magnet synchronous motor," Electrical Machines and Systems (ICEMS), 2011 International Conference on , vol., no., pp.1-5, 20-23 Aug. 2011

[7] Kestelyn, X.; Semail, E.; , "A Vectorial Approach for Generation of Optimal Current References for Multiphase Permanent-Magnet Synchronous Machines in Real Time," Industrial Electronics, IEEE Transactions on , vol.58, no.11, pp.5057-5065, Nov. 2011

[8] D. Casadei, D. Dujic, E. Levi, G. Serra, A. Tani, L. Zarri, "General Modulation Strategy for Seven-Phase Inverters with Independent Control of Multiple Voltage Space Vectors," IEEE Trans. on Industrial Electronics, Vol. 23, No. 2, May 2008, pp.1921-1932. 


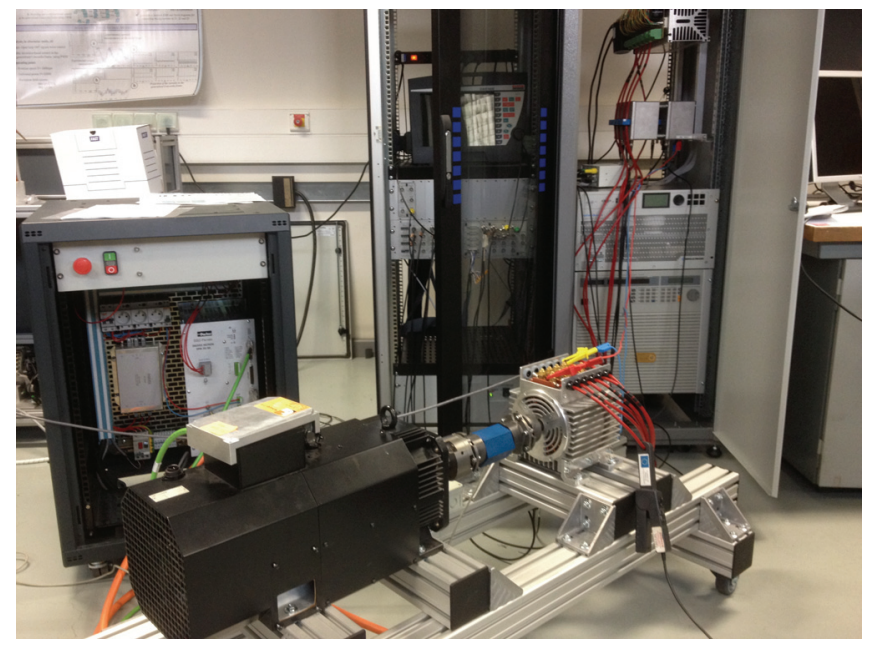

Fig. 3. Snapshot of the experimental test bed

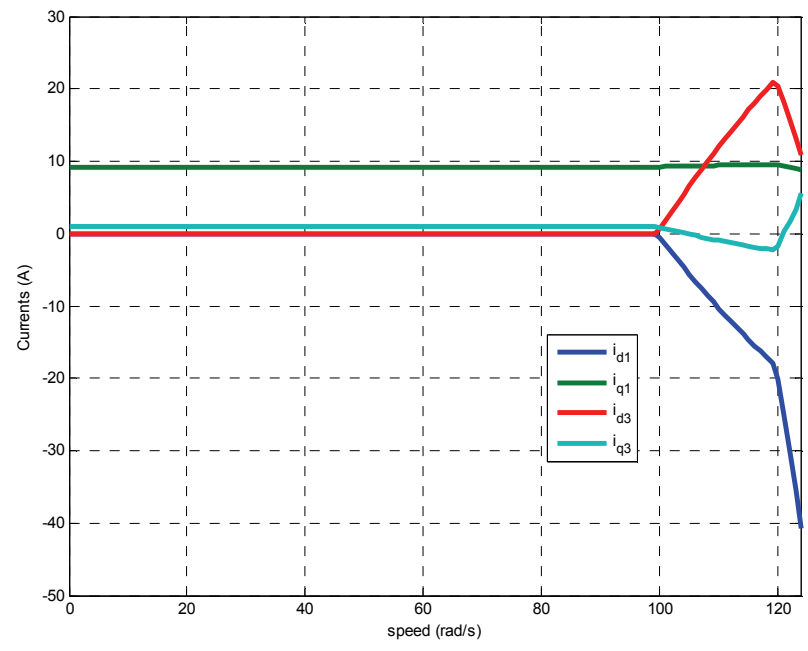

Fig. 5. $d q$ currents versus speed for a torque equals to 1 N.m

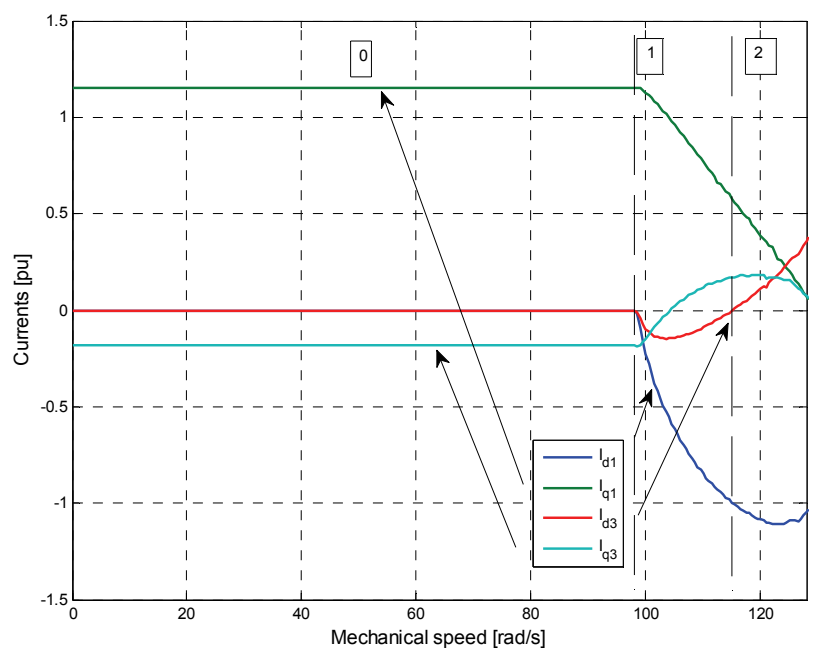

Fig. 7. $d q$ current references versus speed for a maximum torque and maximum phase current

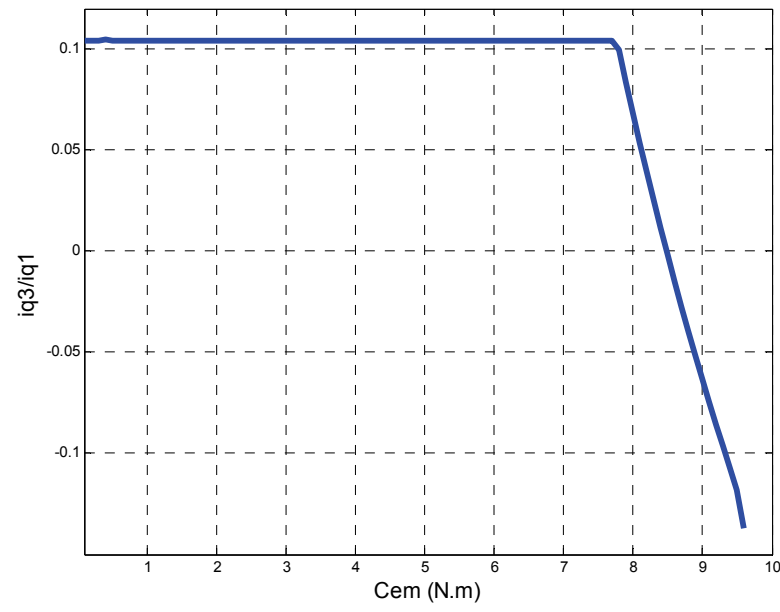

Fig. 4. $\left(i_{3 q} / i_{1 q}\right)$ versus torque below the base speed (at $\left.50 \mathrm{rad} / \mathrm{s}\right) \mathrm{S}$

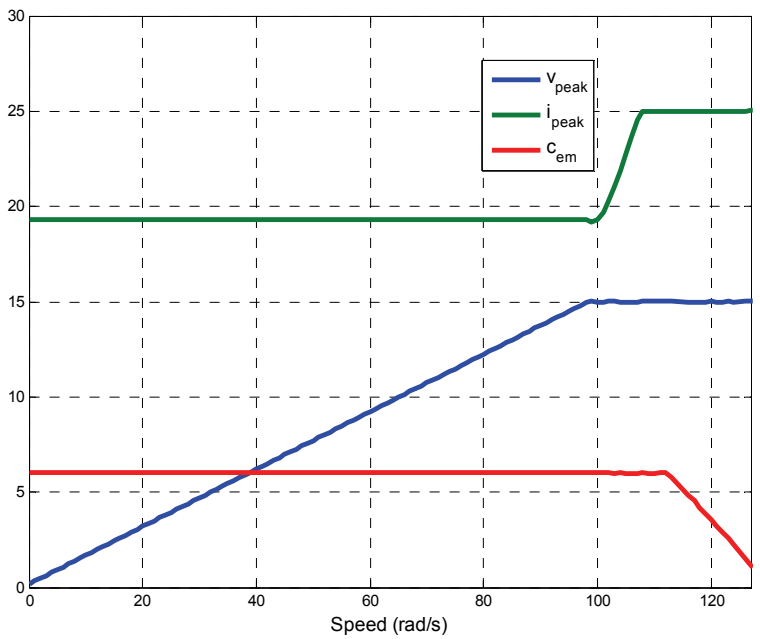

Fig. 6. Voltage phase peak, current phase peak and torque versus speed for a torque refrence equals to $6 \mathrm{~N} . \mathrm{m}$ 


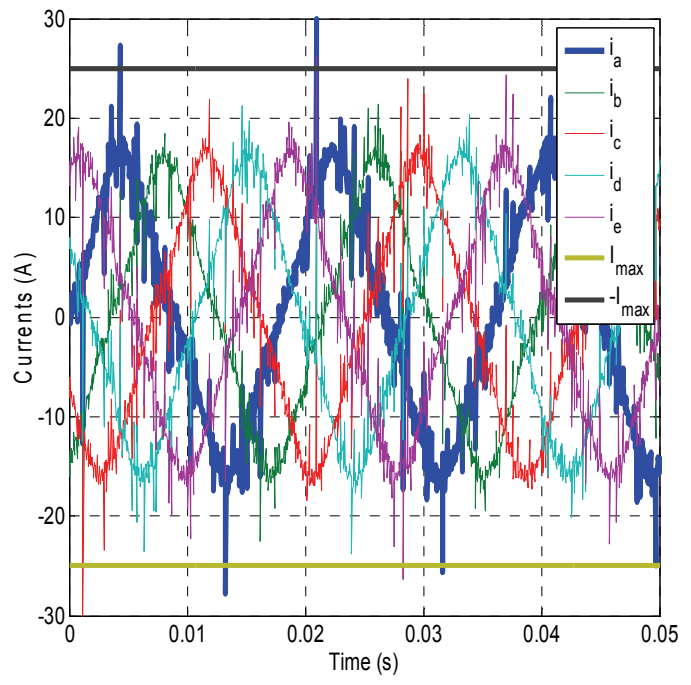

Fig. 8. Experimental phase currents for 5 N.m at $50 \mathrm{rad} / \mathrm{s}$

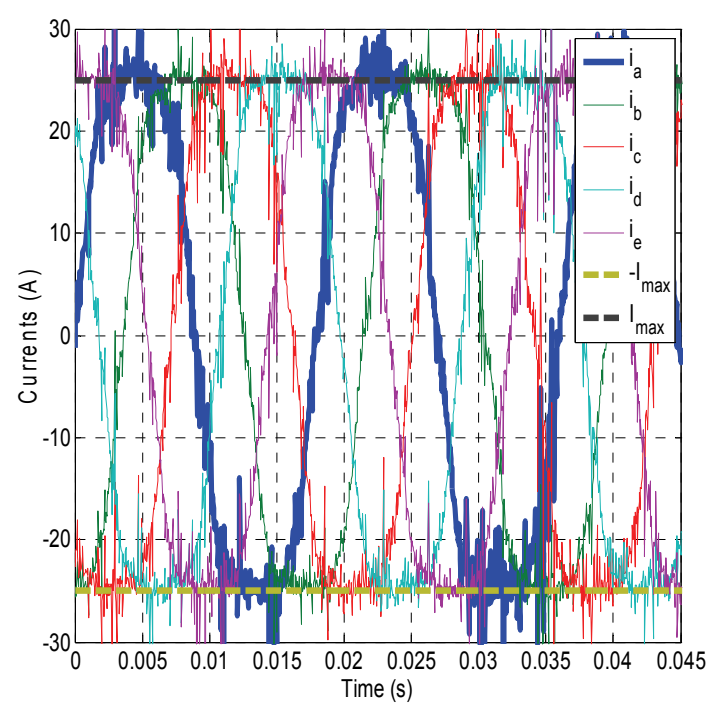

Fig. 10. Experimental phase currents for 9.6 N.m at $50 \mathrm{rad} / \mathrm{s}$

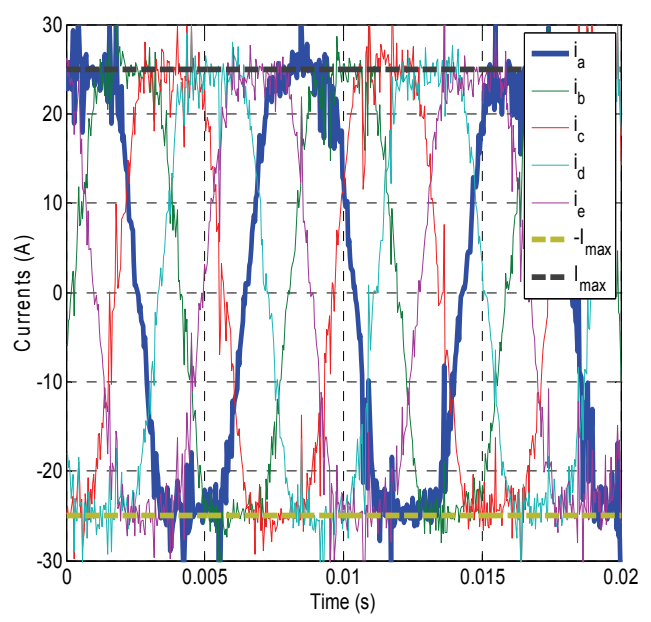

Fig. 12. Experimental phase currents for 5 N.m at $115 \mathrm{rad} / \mathrm{s}$

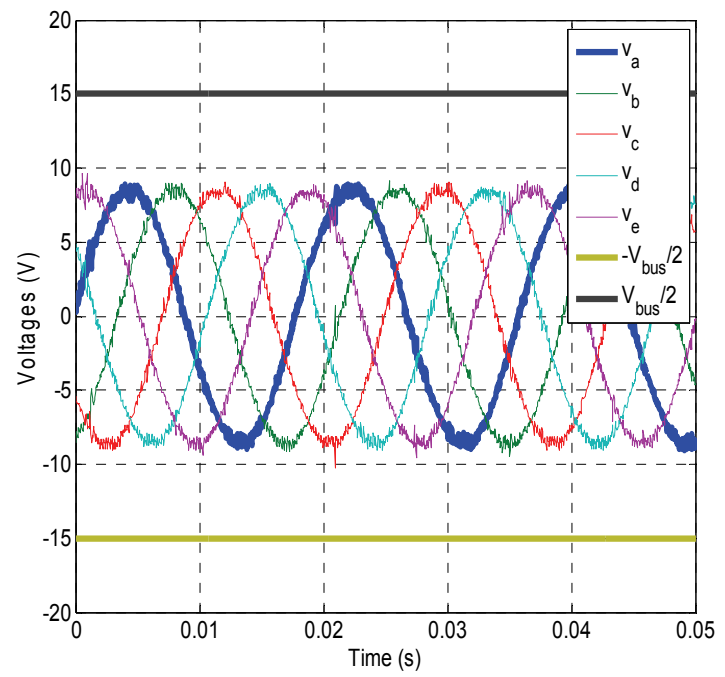

Fig. 9. Experimental phase voltages for 5 N.m at $50 \mathrm{rad} / \mathrm{s}$

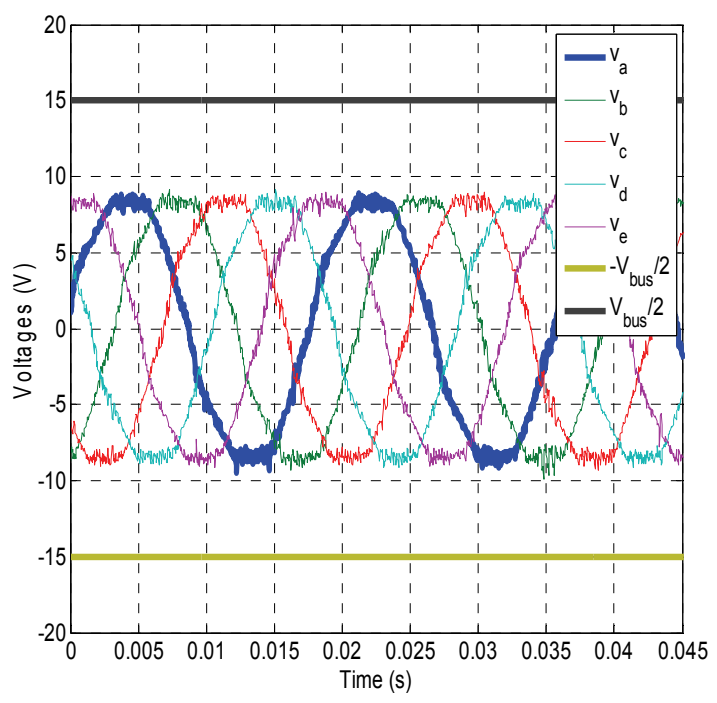

Fig. 11. Experimental phase voltages for 9.6 N.m at $50 \mathrm{rad} / \mathrm{s}$

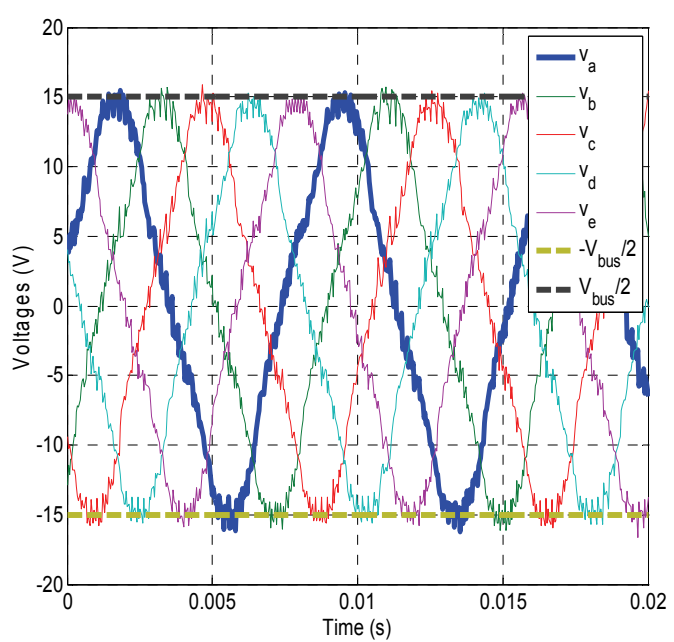

Fig. 13. Experimental phase voltages for 5 N.m at $115 \mathrm{rad} / \mathrm{s}$ 